\title{
COMMENTARY
}

\section{The role of natural killer cells in the pathogenesis of sepsis: the ongoing enigma}

\author{
Julia Bohannon, Yin Guo and Edward R Sherwood* \\ See related research by Souza-Fonseca-Guimaraes et al., http://ccforum.com/content/16/5/R206
}

\begin{abstract}
The study by Souza-Fonseca-Guimaraes and colleagues in the previous issue of Critical Care shows several alterations in blood natural killer (NK) characteristics during human sepsis and systemic inflammatory response syndrome, including changes in NK cell numbers, Toll-like receptor (TLR) expression, and responsiveness to TLR agonists. This paper advances our knowledge of NK cell biology during sepsis and provides the background for future investigations.
\end{abstract}

The paper by Souza-Fonseca-Guimaraes and colleagues [1] in the previous issue of Critical Care expands our understanding of natural killer (NK) cell biology during human sepsis. This is the first study to describe Toll-like receptor (TLR) protein expression by human NK cells. This paper also extends previous reports by localizing TLR to specific cellular compartments and documenting differences in compartmental expression between normal volunteers and patients with sepsis or systemic inflammatory response syndrome (SIRS). The authors report that naive human NK cells express both TLR2 and TLR4, primarily intracellularly, and that intracellular TLR2 expression is upregulated among CD56 $6^{\mathrm{dim}}$ NK cells during sepsis and SIRS. An increase in the percentage of NK cells expressing surface TLR4 was observed during SIRS but not sepsis, whereas increased intracellular TLR4 expression was observed during both conditions. The authors postulate that surface TLR4 expression could be used to discriminate patients with SIRS from patients with sepsis. However, that contention requires further investigation because the SIRS population evaluated in

*Correspondence: edward.r.sherwood@vanderbilt.edu

Department of Anesthesiology, Vanderbilt University Medical Center,

Basic Research Division, MCN T-4202, 1161 Medical Center Drive, Nashville, TN 37232, USA this investigation is not well defined. Patients with SIRS were described as demonstrating clinical criteria for SIRS with an absence of infection. However, little clinical information is provided for the SIRS group to define the etiology of the acute inflammatory syndrome in that population. In addition, a relatively small number of subjects were enrolled in this study, and this makes it difficult to establish a surface TLR4 expression threshold that can be used to define the SIRS population. Nevertheless, their novel observation provides a starting point for further study.

This report documents attenuated ex vivo interferon gamma (IFNY) production by NK cells from patients with sepsis or SIRS in response to TLR agonists and NK cellactivating cytokines. This finding is consistent with the 'endotoxin tolerant' phenotype that has been described in patients with sepsis $[2,3]$ but extends prior observations by showing specific alterations among NK cells. However, the functional significance of this observation remains to be determined. Is this a protective mechanism that acts to decrease NK cell-mediated injury during sepsis? Alternatively, diminished NK cell function may impair the host response to ongoing and secondary infections. Impaired IFNY production is considered a hallmark of sepsis-induced immunosuppression $[4,5]$, yet the contribution of NK cell dysfunction to sepsis-induced immunosuppression has not been determined and deserves further consideration.

The decrease in blood NK cell numbers reported in this article is consistent with previous observations. Boomer and colleagues [6] demonstrated decreased numbers of NK cells in the blood of patients with sepsis. A decline in blood NK cell numbers has also been documented in septic mice [7]. The mechanisms underlying the drop in blood NK cell numbers are not completely clear, but recent studies have documented that NK cells migrate from blood into sites of infection early during the course of sepsis in mice $[7,8]$. NK cell apoptosis is another likely mechanism [9]. Regardless of the mechanism, this study supports the contention that both CD56 ${ }^{\text {dim }}$ and CD $56^{\text {bright }}$ NK cells become activated early during the course of 
sepsis. However, it is currently unclear whether both NK cell subsets play a functional role in the pathogenesis of human sepsis. Owing to differences among NK cell populations in humans and mice, that question may be difficult to answer. Nevertheless, Marquardt and colleagues [10] have postulated that murine CXCR3 ${ }^{+}$NK cells are functionally similar to CD56 bright $^{\text {NK }}$ cells in humans. Recent studies show that $\mathrm{CXCR}^{+}{ }^{+} \mathrm{NK}$ cells migrate to sites of infection and that blockade or ablation of CXCR3 provides functional improvement in experimental models of sepsis [8]. That observation supports a role for CD56 ${ }^{\text {bright }}$ NK cells in the pathogenesis of murine sepsis. However, it is unclear whether those findings will extrapolate to humans. The analysis of NK cell functions during human sepsis is further complicated by the inability to access NK cells in tissues other than blood. It is possible, even likely, that the functions of blood NK cells differ from those of their tissue counterparts during sepsis and that analysis of blood NK cells provides an incomplete picture of global NK cell activity.

A major question that remains is whether NK cell activation is deleterious or beneficial (or both) during sepsis. In a review article published in Molecular Medicine earlier this year, Souza-Fonseca-Guimaraes and colleagues [11] outlined the beneficial and deleterious effects of NK cell activation during bacterial infection. The authors acknowledged evidence that NK cells facilitate the innate immune response against bacterial infection. However, NK cells also appear to mediate tissue damage during the acute phase of sepsis. Therefore, it is likely that NK cells have beneficial and deleterious effects during systemic infection, and this will intensify the challenge of manipulating NK cell functions for therapeutic benefit during sepsis.

\section{Abbreviations}

IFNy, interferon gamma; NK, natural killer; SIRS, systemic inflammatory response syndrome; TLR, Toll-like receptor.
Competing interests

The authors declare that they have no competing interests.

Published: 11 December 2012

\section{References}

1. Souza-Fonseca-Guimaraes F, Parlato M, Philippart F, Misset B, Cavaillon JM, Adib-Conquy M, Study Group C: Toll-like receptors expression and interferon-gamma production by NK cells in human sepsis. Crit Care 2012, 16:R206.

2. Oberholzer A, Harter L, Feilner A, Steckholzer U, Trentz O, Ertel W: Differential effect of caspase inhibition on proinflammatory cytokine release in septic patients. Shock 2000, 14:253-257; discussion 7-8.

3. Biswas SK, Lopez-Collazo E: Endotoxin tolerance: new mechanisms, molecules and clinical significance. Trends Immunol 2009, 30:475-487.

4. Muenzer JT, Davis CG, Chang K, Schmidt RE, Dunne WM, Coopersmith CM, Hotchkiss RS: Characterization and modulation of the immunosuppressive phase of sepsis. Infect Immun 2010, 78:1582-1592.

5. Flohe SB, Agrawal H, Flohe S, Rani M, Bangen JM, Schade FU: Diversity of interferon gamma and granulocyte-macrophage colony-stimulating factor in restoring immune dysfunction of dendritic cells and macrophages during polymicrobial sepsis. Mol Med 2008, 14:247-256.

6. Boomer JS, Shuherk-Shaffer J, Hotchkiss RS, Green JM: A prospective analysis of lymphocyte phenotype and function over the course of acute sepsis. Crit Care 2012, 16:R112.

7. Etogo AO, Nunez J, Lin CY, Toliver-Kinsky TE, Sherwood ER: NK but not CD1restricted NKT cells facilitate systemic inflammation during polymicrobial intra-abdominal sepsis. J Immunol 2008, 180:6334-6345.

8. Herzig DS, Driver BR, Fang G, Toliver-Kinsky TE, Shute EN, Sherwood ER: Regulation of lymphocyte trafficking by CXC chemokine receptor 3 during septic shock. Am J Respir Crit Care Med 2012, 185:291-300.

9. McDunn JE, Hotchkiss RS: Leukocyte phenotyping to stratify septic shock patients. Crit Care 2009, 13:127.

10. Marquardt N, Wilk E, Pokoyski C, Schmidt RE, Jacobs R: Murine CXCR3 $+C D 27$ bright NK cells resemble the human CD56bright NK-cell population. Eur I Immunol 2010, 40:1428-1439.

11. Souza-Fonseca-Guimaraes F, Adib-Conquy M, Cavaillon JM: Natural killer (NK) cells in antibacterial innate immunity: angels or devils? Mol Med 2012, 18:270-285.

doi:10.1186/cc11881

Cite this article as: Bohannon J, et al:: The role of natural killer cells in the pathogenesis of sepsis: the ongoing enigma. Critical Care 2012, 16:185. 